\title{
A Note on a Maximum k-Subset Intersection Problem
}

\author{
Eduardo C. Xavier * \\ Institute of Computing \\ University of Campinas - UNICAMP \\ Campinas, SP, Brazil \\ eduardo@ic.unicamp.br
}

March 12, 2012

\begin{abstract}
Consider the following problem which we call Maximum k-Subset Intersection (MSI): Given a collection $\mathcal{C}=\left\{S_{1}, \ldots, S_{m}\right\}$ of $m$ subsets over a finite set of elements $\mathcal{E}=\left\{e_{1}, \ldots, e_{n}\right\}$, and a positive integer $k$, the objective is to select exactly $k$ subsets $S_{j_{1}}, \ldots, S_{j_{k}}$ whose intersection size $\left|S_{j_{1}} \cap \ldots \cap S_{j_{k}}\right|$ is maximum. In [2], Clifford and Popa studied a related problem and left as an open problem the status of the MSI problem. In this paper we show that this problem is hard to approximate.
\end{abstract}

Key Words: Approximation algorithms, Combinatorial problems, Subset Intersection

\section{Introduction}

In this paper we study the following problem: Given a collection $\mathcal{C}=\left\{S_{1}, \ldots, S_{m}\right\}$ of $m$ subsets over a finite set of elements $\mathcal{E}=\left\{e_{1}, \ldots, e_{n}\right\}$, and a positive integer $k$, the objective is to select exactly $k$ subsets $S_{j_{1}}, \ldots, S_{j_{k}}$ from $\mathcal{C}$ whose intersection size $\left|S_{j_{1}} \cap \ldots \cap S_{j_{k}}\right|$ is maximum. We call this problem Maximum k-Subset Intersection (MSI), which was left as an open problem by Clifford and Popa [2].

In this paper we present an inapproximability result for the MSI problem presenting a reduction from the Maximum Edge Biclique ( $M E B$ ) problem. The MEB problem can be stated as follows: Given a bipartite graph $G=\left(V_{1}, V_{2}, E\right)$, the problem is to find a biclique $K_{x, y}$ subgraph of $G$ whose number of edges $x y$ is maximum.

The MEB problem was shown to be NP-hard by Peteers [5]. Later, Ambuhl et al in [1], proved that the MEB problem does not admit a $1 / N^{\epsilon^{\prime}}$ approximation, where $\epsilon^{\prime}$ is a constant and $N$ is the number of vertices, under the standard assumption that SAT has no probabilistic algorithm that runs in time $2^{n^{\epsilon}}$, where $n$ is the instance size and $\epsilon>0$ can be made arbitrarily close to 0 . They showed the following result:

Theorem 1 (Ambuhl et al [1]) Let $\epsilon>0$ be an arbitrarily small constant. Assume that SAT does not have a probabilistic algorithm that decides whether a given instance of size $n$ is satisfiable in time $2^{n^{\epsilon}}$. Then there is no polynomial (possibly randomized) algorithm for Maximum Edge Biclique that achieves an approximation ratio of $1 / N^{\epsilon^{\prime}}$ on graphs of size $N$, where $\epsilon^{\prime}$ depends only on $\epsilon$.

In this work we show an inapproximability result for the MSI problem using the inapproximability result of Theorem 1.

\footnotetext{
${ }^{*}$ Supported by $\mathrm{CNPq}$
} 
The MEB problem has applications in community detection [3] and in bioinformatics [4], among others. The biclustering problems involved in such applications can also be tackled as a MSI problem. Generally, we have in such applications a set of individuals/genes and associated interests/conditions. The main objective is to find a set of individuals/genes with the largest number of interests/conditions in common.

In Section 2 we present a Turing reduction showing the hardness of the MSI problem, and in Section 3 we prove the inapproximability of the MSI problem by showing that if there is an $\alpha$-approximation algorithm for the MSI problem, then there is also an $\alpha$-approximation algorithm for the MEB problem.

\section{Hardness Result}

In this section we present a Turing reduction from the MEB problem to the MSI problem, by presenting a polynomial time algorithm that can be used to solve the MEB problem if the MSI problem is solvable in polynomial time.

\section{Theorem 2 MSI is NP-hard.}

Proof. Let $G=\left(V_{1}, V_{2}, E\right)$ be an instance for the MEB problem, where $V_{1}=\left\{v_{1}, \ldots, v_{n_{1}}\right\}$ and $V_{2}=$ $\left\{u_{1}, \ldots, u_{n_{2}}\right\}$. Create an instance for the MSI problem as follows: let the set of elements be the set $V_{2}$, i.e, $\mathcal{E}=V_{2}$, and for each vertex $v_{i} \in V_{1}$ create a set $v_{i}=\left\{u_{j} \in V_{2}:\left(v_{i}, u_{j}\right) \in E\right\}$, i.e, this set contains all vertices of $V_{2}$ that are adjacent to $v_{i}$. The collection of subsets is $\mathcal{C}=\left\{v_{1}, \ldots, v_{n_{1}}\right\}$.

Considering the construction above, we claim that for any given biclique subgraph $K_{x, y}$ of $G$, there are $x$ subsets in the corresponding instance of the MSI problem such that their intersection size is at least $y$. Let $V_{1}^{\prime} \subseteq V_{1}$ and $V_{2}^{\prime} \subseteq V_{2}$ be the vertices of the biclique $K_{x, y}$. Since every vertex in $V_{1}^{\prime}$ is adjacent to all vertices in $V_{2}^{\prime}$, then all vertices of $V_{2}^{\prime}$ will belong to each subset corresponding to each vertex of $V_{1}^{\prime}$. The intersection of these subsets contains $V_{2}^{\prime}$.

On the other hand, we claim that if we find $k$ subsets $V_{1}^{\prime}=\left\{v_{1}^{\prime}, \ldots, v_{k}^{\prime}\right\}$ of maximum intersection $v_{1}^{\prime} \cap$ $\ldots \cap v_{k}^{\prime}=V_{2}^{\prime} \subseteq V_{2}$, then there is a biclique subgraph in $G$ with $k\left|V_{2}^{\prime}\right|$ edges. From the construction of the MSI instance, every vertex $v_{i}^{\prime}$ is adjacent to all vertices in $V_{2}^{\prime}$. Then the induced subgraph given by the corresponding vertices in $V_{1}^{\prime}$ and $V_{2}^{\prime}$ form a biclique of size $k\left|V_{2}^{\prime}\right|$.

Suppose there is a polynomial time algorithm $\mathcal{A}(\mathcal{C}, k, \mathcal{E})$ that solves the MSI problem, and returns $\left(\mathcal{C}^{\prime}, I\right)$, where $\mathcal{C}^{\prime} \subset \mathcal{C}$ contains $k$ subsets, and $I$ contains the elements of the intersection of these subsets. Then Algorithm 1 solves the MEB problem.

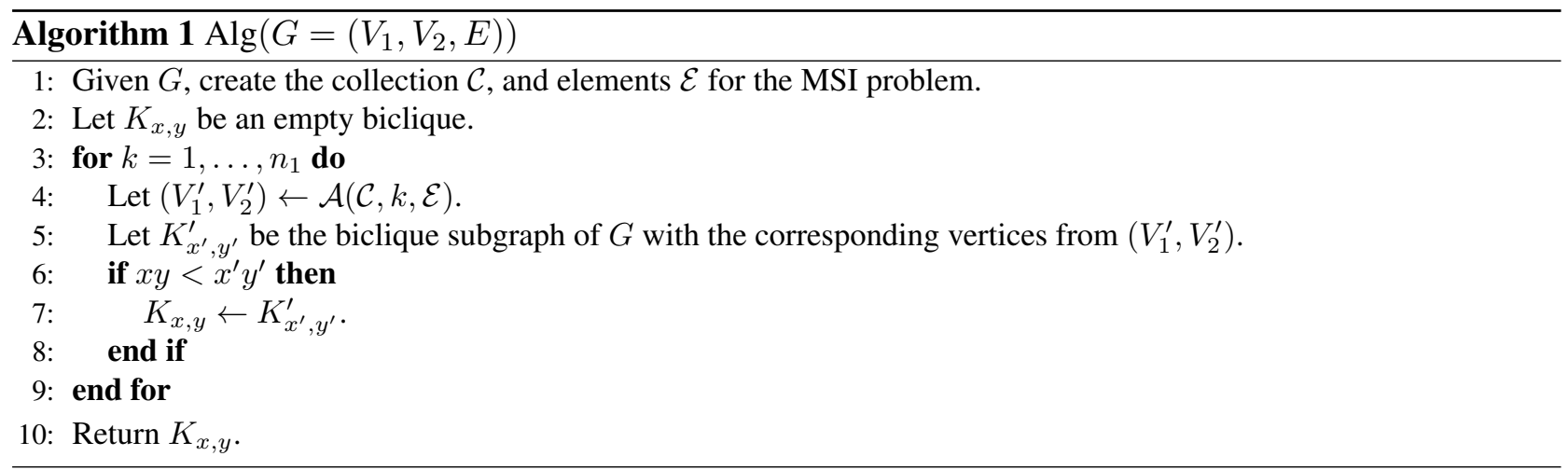

Let $K_{x^{*}, y^{*}}^{*}$ be an optimal solution for the MEB problem. We know that when we run $\mathcal{A}\left(\mathcal{C}, x^{*}, \mathcal{E}\right)$, the algorithm will return a solution corresponding to vertices that form a biclique subgraph of $G$ with at least $x^{*} y^{*}$ edges. Since the algorithm tries all values of $k=1, \ldots, n_{1}$, and returns the biclique with maximum number of edges, it will return an optimal solution. 


\section{Inapproximability Result}

In this section we show that if there is an $\alpha$-approximation algorithm $\mathcal{A}(\mathcal{C}, k, \mathcal{E})$ for the MSI problem then we can construct another algorithm $\mathcal{A}^{\prime}$ which is an $\alpha$-approximation algorithm for the MEB problem.

Lemma 3 Let $\mathcal{A}$ be an $\alpha$-approximation algorithm for the MSI problem. Then there is an $\alpha$-approximation algorithm $\mathcal{A}^{\prime}$ for the MEB problem.

Proof. Let $G=\left(V_{1}, V_{2}, E\right)$ be an instance of the MEB problem, where $n_{1}=\left|V_{1}\right|$ and $n_{2}=\left|V_{2}\right|$. We construct an instance for the MSI problem as was done in Theorem 2.

Suppose that $K_{x, y}$ is a maximum edge biclique of $G$. If we construct an instance for the MSI problem as stated above, and run $\mathcal{A}(\mathcal{C}, x, \mathcal{E})$ we know that the algorithm is going to find $x$ subsets $v_{i_{1}}, \ldots, v_{i_{x}}$, whose intersection size is at least $\alpha y$. Notice that the vertices $v_{i_{1}}, \ldots, v_{i_{x}}$ from $V_{1}$ and the vertices in the corresponding intersection of their subsets, form a biclique with at least $\alpha x y$ edges.

Suppose we run $\mathcal{A}(\mathcal{C}, k, \mathcal{E})$, for $k=1, \ldots, n_{1}$. We can then find the solution $v_{i_{1}}^{\prime}, \ldots, v_{i_{k^{\prime}}}^{\prime}$ that maximizes the value $k^{\prime} T$ where $T=\left|v_{i_{1}}^{\prime} \cap \ldots \cap v_{i_{k}^{\prime}}^{\prime}\right|$, among all these executions of the algorithm. Notice that the corresponding vertices $v_{i_{1}}^{\prime}, \ldots, v_{i_{k^{\prime}}}^{\prime}$ from $V_{1}$ and vertices in $v_{i_{1}}^{\prime} \cap \ldots \cap v_{i_{k^{\prime}}}^{\prime}$ from $V_{2}$, form a biclique of size $k^{\prime} T \geqslant \alpha x y$. Then we have an $\alpha$-approximation solution for the given instance $G$ of the MEB problem.

Using Theorem 1 and Lemma 3 we have the following result.

Theorem 4 Let $\epsilon>0$ be an arbitrarily small constant. Assume that SAT does not have a probabilistic algorithm that decides whether a given instance of size $n$ is satisfiable in time $2^{n^{\epsilon}}$. Then there is no polynomial time algorithm for the Maximum $k$-Subset Intersection problem that achieves an approximation ratio of $1 / N^{\epsilon^{\prime}}$ where $N$ is the size of the instance, and $\epsilon^{\prime}$ depends only on $\epsilon$.

\section{References}

[1] Christoph Ambühl, Monaldo Mastrolilli, and Ola Svensson. Inapproximability results for maximum edge biclique, minimum linear arrangement, and sparsest cut. SIAM J. Comput., 40(2):567-596, 2011.

[2] Raphaël Clifford and Alexandru Popa. Maximum subset intersection. Inf. Process. Lett., 111(7):323-325, 2011.

[3] Santo Fortunato. Community detection in graphs. Physics Reports, 486(3-5):75-174, 2010.

[4] Sushmita Mitra and Haider Banka. Multi-objective evolutionary biclustering of gene expression data. Pattern Recognition, 39(12):2464-2477, 2006.

[5] René Peeters. The maximum edge biclique problem is np-complete. Discrete Applied Mathematics, 131(3):651-654, 2003. 